\title{
A complete response to mFOLFOX6 and panitumumab chemotherapy in advanced stage rectal adenocarcinoma: a case report
}

\author{
Hiromichi Sonoda ${ }^{1 *}$, Tomoharu Shimizu$^{1}$, Eiji Mekata', Yoshihiro Endo ${ }^{1}$, Mitsuaki Ishida $^{2}$ and Tohru Tani ${ }^{1}$
}

\begin{abstract}
Background: Pathological complete remission of advanced stage rectal adenocarcinoma by chemotherapy alone is rare. A case of advanced stage, low-lying rectal adenocarcinoma in which a complete response to treatment was obtained with mFOLFOX6 and panitumumab (Pmab) is reported.

Case presentation: A 53-year-old man was referred to Shiga University of Medical Science hospital Shiga, Japan, complaining of bloody stool. Gastrointestinal endoscopy was performed, and advanced stage rectal adenocarcinoma was diagnosed. Computed tomography (CT) revealed regional lymph node metastases in the mesorectum.

Neoadjuvant chemotherapy (NAC) with mFOLFOX6 and Pmab was planned.

Endoscopy following four courses of chemotherapy revealed that the rectal cancer had been markedly reduced, and the results of biopsies of the rectal tumor were negative for cancer. On $C T$, the mesorectal lymph node metastases had disappeared. Total intersphincteric resection (ISR) with a handsewn coloanal anastomosis was performed. Histological examination showed a complete response to MFOLFOX6 and Pmab in advanced stage rectal cancer.
\end{abstract}

Conclusion: The result seen in this case suggests that short-term NAC with mFOLFOX6 and Pmab was effective for low-lying rectal adenocarcinoma.

Keywords: Neoadjuvant chemotherapy, Rectal cancer, Panitumumab

\section{Background}

In Western countries, the standard treatment for advanced stage rectal cancer is neoadjuvant chemoradiotherapy (CRT) followed by surgery [1]. On the other hand, curative surgery including lateral lymph node dissection followed by adjuvant chemotherapy is the standard treatment for advanced rectal cancer in Japan [2]. Recently, neoadjuvant CRT followed by anus-preserving surgery has also been reported in Japan [3]. However, it was reported that postoperative anal function was decreased when the effect of preoperative CRT was strong in patients treated with intersphincteric resection (ISR) [3]. Use of panitumumab (Pmab) and cetuximab (Cmab), anti-endothelial growth factor receptor (EGFR) antibodies, has resulted in earlier tumor shrinkage for K-ras wild type metastatic colorectal cancer [4]. The case of a 53-year-old man with stage III

\footnotetext{
* Correspondence: hirosono@belle.shiga-med.ac.jp

'Department of Surgery, Shiga University of Medical Science,

Seta Tsukinowa-cho, Otsu, Shiga 520-2192, Japan

Full list of author information is available at the end of the article
}

low rectal cancer who had a complete response to neoadjuvant oxaliplatin, 5-fluorouracil (5-FU) and l-folinic acid (mFOLFOX6) and Pmab chemotherapy without concurrent radiotherapy is reported.

\section{Case presentation}

A 53-year-old man was referred to Shiga University of Medical Science hospital, Shiga, Japan, complaining of bloody stool. The patient was diagnosed as having a $3 \mathrm{~cm}$ in length, type $2 \mathrm{~K}$-ras wild type rectal cancer, $2 \mathrm{~cm}$ from the anal verge (Figure 1a) that invaded to the dentate line (Figure 1b) on screening colonoscopy. Computed tomography $(\mathrm{CT})$ revealed rectal wall thickening and a regional lymph node metastasis in the mesorectum (Figure 2a). Advanced stage, low-lying rectal cancer was diagnosed. We usually perform abdominoperineal resection (APR) for advanced rectal cancer located in the anal canal as in this case. However, the patient was not willing to undergo APR.

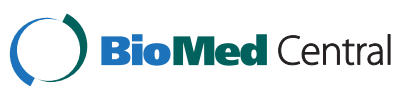




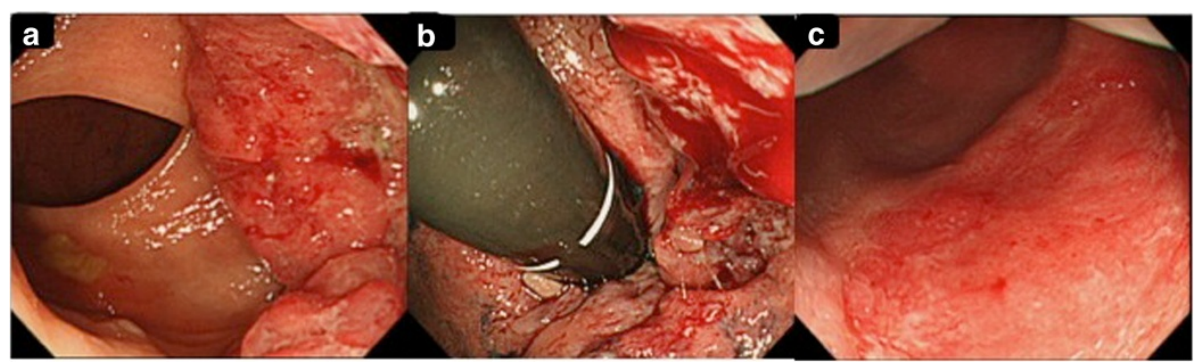

Figure 1 Colonoscopy images. (a) Colonoscopy imaging shows a $3 \mathrm{~cm}$ in length, type 2 rectal cancer (b) that invades to the dentate line. (c) Repeated colonoscopy after chemotherapy shows an excellent response with only injected mucosal scar in the area of the previously identified rectal cancer.

Previously, Canda et al. reported that neoadjuvant CRT may adversely affect anorectal function [5]. It has also been reported that $\mathrm{Cmab}$ and Pmab-containing chemotherapy enables early tumor shrinkage [4]. For these reasons, neoadjuvant chemotherapy (NAC) with mFOLFOX6 and Pmab was performed every 2 weeks with the patient's written, informed consent. The patient received a total of four cycles of chemotherapy over 2 months.

Repeated colonoscopy after chemotherapy showed an excellent response with only injected mucosal scar in the area of the previously identified rectal cancer (Figure 1c). The results of the biopsies were negative for cancer cells. Additionally, CT scan demonstrated no rectal wall thickening and no mesorectal lymph node metastasis (Figure $2 \mathrm{~b}$ ). The patient underwent total ISR with a handsewn coloanal anastomosis, total mesorectal and bilateral pelvic lymph node dissection, and temporary loop ileostomy 3 weeks after completion of NAC. No residual carcinoma was found (Figure 3), 12 noninvolved lymph nodes were identified, and all margins were negative for cancer on histologic examination.

5-FU-based adjuvant chemotherapy after neoadjuvant CRT followed by surgery is the standard treatment for clinical stage III rectal cancer [1]. For this reason, the patient received adjuvant chemotherapy with tegafur-uracil (UFT) and leucovorin administration for 3 months after surgery. Three months after surgery, the patient is alive without evidence of recurrence of cancer.

\section{Discussion}

Preoperative 5-FU-based concurrent CRT followed by surgery is the standard strategy for advanced rectal cancer in Western countries [1]. However, it has been suggested that preoperative CRT is strongly associated with postoperative complications, including anastomotic leakage [6] and poor anal function, after low anterior resection and ISR [3]. It has been reported that preoperative CRT also increases late complications such as sexual and voiding dysfunctions [7], intestinal and defecation problems [8], and secondary carcinogenesis [9]. All of these have recently become issues in Western countries.

Recently, new anticancer agents have markedly improved the response rate and prognosis of unresectable and recurrent colorectal cancer. Oxaliplatin-based combination chemotherapy improves survival of colorectal cancer patients in the metastatic and adjuvant settings [10-12]. Oxaliplatin-containing 'induction chemotherapy' before CRT is associated with an objective response rate of up to $88 \%$ and rapid symptomatic improvement $[13,14]$. These regimens may also convert unresectable liver metastases to resectable ones $[15,16]$. It has been reported that bevacizumab (Bmab), an anti-vascular endothelial growth factor (VEGF) receptor antagonist, added to oxaliplatin-based chemotherapy, capecitabine plus oxaliplatin (XELOX), resulted in a high response rate for patients with colorectal liver metastases [17]. In Japan, a multicenter phase II trial

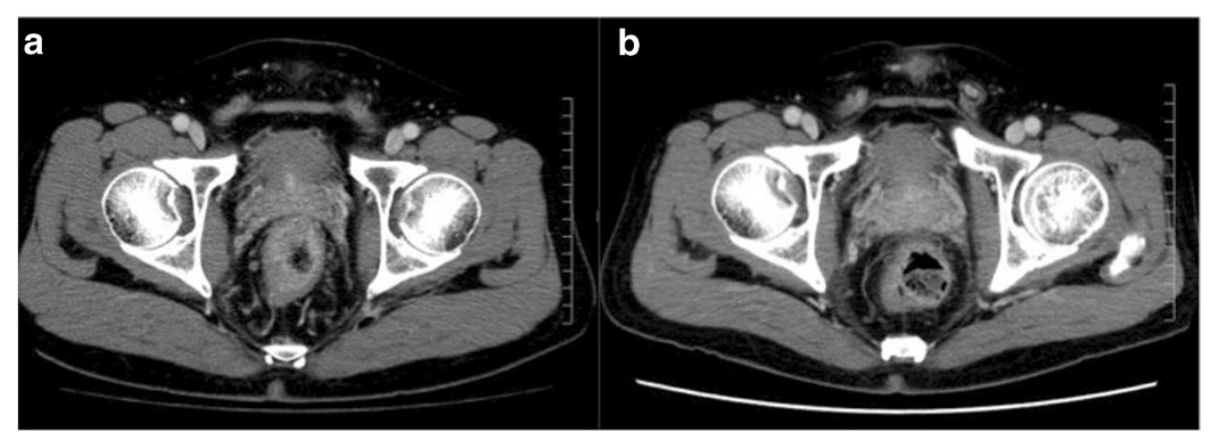

Figure 2 Computed tomography (CT) images. (a) $\subset$ T imaging reveals rectal wall thickening and a regional lymph node metastasis in the mesorectum. (b) $\subset$ scan after chemotherapy demonstrates no rectal wall thickening and no mesorectal lymph node metastasis. $\subset$, computed tomography. 


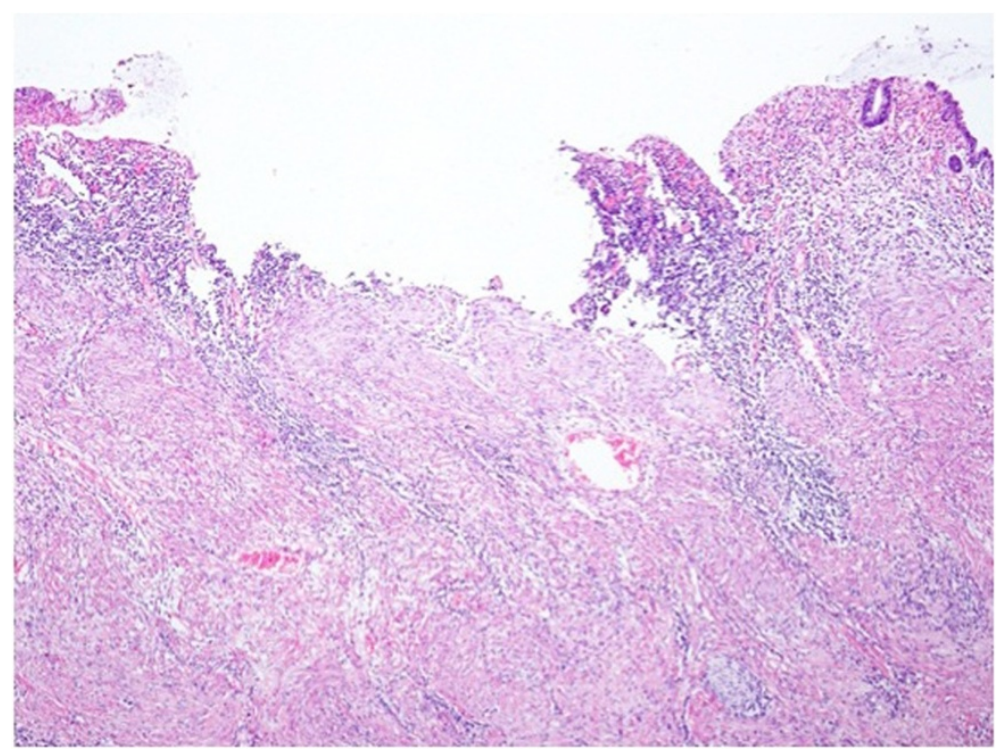

Figure 3 No residual carcinoma is found on histologic examination.

of NAC with XELOX plus Bmab for locally advanced rectal cancer is now ongoing [18]. In addition, two retrospective studies have shown that the early dimensional reduction of target lesions after 6 weeks from the beginning of treatment is an indicator of sensitivity to Cmab [19,20]. Pmab, a fully human immunoglobulin G (IgG) anti-EGFR antibody, has been considered equally effective in patients with $K$-ras wild type refractory metastatic colorectal cancer. Cmab must be administered every week, while Pmab can be administered every 2 weeks.

In the neoadjuvant setting, surgery must be delayed for at least 1 month after the last Bmab-containing chemotherapy. However, it is not necessary to delay surgery after anti-EGFR-containing chemotherapy. Because of these reasons, we considered that preoperative MFOLFOX6 and Pmab chemotherapy should be effective for this case. Recently, Li et al. reported a case of advanced rectal cancer demonstrating a pathologic complete response after NAC with six cycles of FOLFOX7 [21]. This case is the first report in the English literature from an Asian country demonstrating a pathological complete response after NAC in a patient with low-lying advanced rectal cancer. In the present case, NAC was given for four cycles, but the appropriate period of NAC administration has not been determined. However, a pilot study demonstrated clear downstaging of primary colon cancer with only three cycles of NAC [22]. Another report showed that detection, by week 2 magnetic resonance imaging, of tumor shrinkage $>10 \%$ in response to therapy with Cmab or Pmab for metastatic colorectal cancer represents an early indicator of clinical outcome because it is predictive of the prolongation of progression-free survival and overall survival [4]. We thought that if NAC was not effective, the patient would not be able to receive curative surgery because of disease progression. Therefore, we evaluated the efficacy of NAC after a short course (four cycles) of chemotherapy. We then decided to perform surgery because of the excellent response to NAC. The present case suggests that Pmab is a good candidate for NAC because of its earlier drug response. We consider NAC is a promising preoperative treatment for locally advanced rectal cancer instead of neoadjuvant CRT. However, there are no data about whether NAC with or without concurrent radiotherapy is effective against advanced rectal cancer. Further studies are needed.

\section{Conclusions}

This case suggests that mFOLFOX6 and Pmab chemotherapy without irradiation may be an alternative therapy for patients with low-lying rectal cancer.

\section{Consent}

Written informed consent was obtained from the patient for publication of this case report and any accompanying images. A copy of the written consent is available for review by the Editor-in-Chief of this journal.

\section{Abbreviations}

5-FU: 5-Fluorouracil; APR: Abdominoperineal resection; Bmab: Bevacizumab; Cmab: Cetuximab; CRT: Chemoradiotherapy; CT: Computed tomography; EGFR: Endothelial growth factor receptor; IgG: Immunoglobulin G; ISR: Intersphincteric resection; MRI: Magnetic resonance imaging; NAC: Neoadjuvant chemotherapy; Pmab: Panitumumab; UFT: Tegafur-uracil; VEGF: Vascular endothelial growth factor; XELOX: Capecitabine plus oxaliplatin.

\section{Competing interests}

The authors declare that they have no competing interests. 


\section{Authors' contributions}

HS drafted the manuscript. TS, EM, YE and TT helped to draft the manuscript. MI created Figure 3. All authors read and approved the final manuscript.

\section{Author details}

${ }^{1}$ Department of Surgery, Shiga University of Medical Science, Seta Tsukinowa-cho, Otsu, Shiga 520-2192, Japan. ²Department of Clinical Laboratory Medicine, Shiga University of Medical Science, Seta Tsukinowa-cho, Otsu, Shiga 520-2192, Japan.

Received: 26 April 2013 Accepted: 15 March 2014

Published: 26 March 2014

\section{References}

1. National Comprehensive Cancer Network (NCCN): NCCN Clinical Practice Guidelines in Oncology: Rectal Cancer. Volume Version 1. Fort Washington, PA: NCCN; 2013.

2. Watanabe T, Itabashi M, Shimada Y, Tanaka S, Ito Y, Ajioka Y, Hamaguchi T, Hyodo I, Igarashi M, Ishida H, Ishiguro M, Kanemitsu Y, Kokudo N, Muro K, Ochiai A, Oguchi M, Ohkura Y, Saito Y, Sakai Y, Ueno H, Yoshino T, Fujimori T, Koinuma N, Morita T, Nishimura G, Sakata Y, Takahashi K, Takiuchi H, Tsuruta O, Yamaguchi T, et al: Japanese Society for Cancer of the Colon and Rectum (JSCCR) guidelines 2010 for the treatment of colorectal cancer. Int J Clin Oncol 2012, 17(1):1-29.

3. Nishizawa Y, Saito N, Fujii S, Ito M, Sugito M, Kobayashi A, Nishizawa Y: Association between anal function and therapeutic effect after preoperative chemoradiotherapy followed by intersphincteric resection. Dig Surg 2012, 29(5):439-445.

4. Ricotta R, Vanzulli A, Moroni M, Colnago B, Oriani M, Nichelatti M, Sarnataro C, Venturini F, Di Bella S, Maiolani M, Giganti MO, Sartore-Bianchi A, Siena S: Magnetic resonance imaging as an early indicator of clinical outcome in patients with metastatic colorectal carcinoma treated with cetuximab or panitumumab. Clin Colorectal Cancer 2013, 12:45-53.

5. Canda AE, Terzi C, Gorken IB, Oztop I, Sokmen S, Fuzun M: Effects of preoperative chemoradiotherapy on anal sphincter functions and quality of life in rectal cancer patients. Int J Colorectal Dis 2010, 25(2):197-204.

6. Park JS, Choi GS, Kim SH, Kim HR, Kim NK, Lee KY, Kang SB, Kim JY, Lee KY, Kim BC, Bae BN, Son GM, Lee SI, Kang H: Multicenter analysis of risk factors for anastomotic leakage after laparoscopic rectal cancer excision: the Korean laparoscopic colorectal surgery study group. Ann Surg 2013, 257:665-671.

7. Marijnen CA, van de Velde CJ, Putter $H$, van den Brink M, Maas CP, Martijn H, Rutten HJ, Wiggers T, Kranenbarg EK, Leer JW, Stiggelbout AM: Impact of short-term preoperative radiotherapy on health-related quality of life and sexual functioning in primary rectal cancer: report of a multicenter randomized trial. J Clin Oncol 1847-1858, 2005:23.

8. Peeters KC, van de Velde CJ, Leer JW, Martijn H, Junggeburt JM, Kranenbarg EK, Steup WH, Wiggers T, Rutten HJ, Marijnen CA: Late side effects of shortcourse preoperative radiotherapy combined with total mesorectal excision for rectal cancer: increased bowel dysfunction in irradiated patients: a Dutch colorectal cancer group study. J Clin Oncol 2005, 23:6199-6206.

9. Wright JD, St Clair CM, Deutsch I, Burke WM, Gorrochurn P, Sun X, Herzog TJ: Pelvic radiotherapy and the risk of secondary leukemia and multiple myeloma. Cancer 2010, 116:2486-2492.

10. André T, Boni C, Mounedji-Boudiaf L, Navarro M, Tabernero J, Hickish T, Topham C, Zaninelli M, Clingan P, Bridgewater J, Tabah-Fisch I, de Gramont A, Multicenter International Study of Oxaliplatin/5-Fluorouracil/Leucovorin in the Adjuvant Treatment of Colon Cancer (MOSAIC) Investigators: Oxaliplatin, fluorouracil, and leucovorin as adjuvant treatment of colon cancer. $N$ Engl J Med 2004, 350:2343-2351

11. Tournigand C, André T, Achille E, Lledo G, Flesh M, Mery-Mignard D, Quinaux E, Couteau C, Buyse M, Ganem G, Landi B, Colin P, Louvet C, de Gramont A: FOLFIRI followed by FOLFOX6 or the reverse sequence in advanced colorectal cancer: a randomized GERCOR study. J Clin Oncol 2004, 22:229-237.

12. Goldberg RM, Sargent DJ, Morton RF, Fuchs CS, Ramanathan RK, Williamson SK, Findlay BP, Pitot HC, Alberts SR: A randomized controlled trial of fluorouracil plus leucovorin, irinotecan, and oxaliplatin combinations in patients with previously untreated metastatic colorectal cancer. J Clin Oncol 2004, 22:23-30.
13. Koeberle D, Burkhard R, von Moos R, Winterhalder R, Hess V, Heitzmann F, Ruhstaller T, Terraciano L, Neuweiler J, Bieri G, Rust C, Toepfer M: Phase II study of capecitabine and oxaliplatin given prior to and concurrently with preoperative pelvic radiotherapy in patients with locally advanced rectal cancer. Br J Cancer 2008, 98:1204-1209.

14. Chua YJ, Barbachano Y, Cunningham D, Oates JR, Brown G, Wotherspoon A Tait D, Massey A, Tebbutt NC, Chau I: Neoadjuvant capecitabine and oxaliplatin before chemoradiotherapy and total mesorectal excision in MRI- defined poor-risk rectal cancer: a phase 2 trial. Lancet Oncol 2010, 11:241-248.

15. Alberts SR, Horvath WL, Sternfeld WC, Goldberg RM, Mahoney MR, Dakhil SR, Levitt R, Rowland K, Nair S, Sargent DJ, Donohue JH: Oxaliplatin, fluorouracil, and leucovorin for patients with unresectable liver-only metastases from colorectal cancer: a North Central Cancer Treatment Group phase II study. J Clin Oncol 2005, 23:9243-9249.

16. Beppu T, Hayashi N, Masuda T, Komori H, Horino K, Hayashi H, Okabe H, Baba Y, Kinoshita K, Akira C, Watanebe M, Takamori H, Baba H: FOLFOX enables high resectability and excellent prognosis for initially unresectable colorectal liver metastases. Anticancer Res 2010, 30:1015-1020.

17. Wong R, Cunningham D, Barbachano Y, Saffery C, Valle J, Hickish T, Mudan S, Brown G, Khan A, Wotherspoon A, Strimpakos AS, Thomas J, Compton S, Chua YJ, Chau I: A multicentre study of capecitabine, oxaliplatin plus bevacizumab as perioperative treatment of patients with poor-risk colorectal liver-only metastases not selected for upfront resection. Ann Oncol 2011, 22(9):2042-2048.

18. Uehara $K$, Ishiguro S, Sakamoto E, Maeda A, Inoue M, Tojima Y, Kobayashi S, Omiya N, Ishizuka N, Nakao A, Goto H, Nagino M: Phase II trial of neoadjuvant chemotherapy with XELOX plus bevacizumab for locally advanced rectal cancer. Jpn J Clin Oncol 2011, 41(8):1041-1044.

19. De Roock W, Piessevaux H, De Schutter J, Janssens M, De Hertogh G, Personeni N, Biesmans B, Van Laethem JL, Peeters M, Humblet $Y$, Van Cutsem E, Tejpar S: KRAS wild-type state predicts survival and is associated to early radiological response in metastatic colorectal cancer treated with cetuximab. Ann Oncol 2008, 19:508-515.

20. Piessevaux H, Buyse M, De Roock W, Prenen H, Schlichting M, Van Cutsem E, Tejpar S: Radiological tumor size decrease at week 6 is a potent predictor of outcome in chemorefractory metastatic colorectal cancer treated with cetuximab (BOND trial). Ann Oncol 2009, 20:1375-1382.

21. Li J, Rose MG, Perkal MF, Chao HH: Pathologic complete response after FOLFOX7 in a locally advanced rectal cancer. J Clin Gastroenterol 2012, 46(1):87-88.

22. Foxtrot Collaborative Group: Feasibility of preoperative chemotherapy for locally advanced, operable colon cancer: the pilot phase of a randomised controlled trial. Lancet Oncol 2012, 13(11):1152-1160.

doi:10.1186/1477-7819-12-63

Cite this article as: Sonoda et al:: A complete response to mFOLFOX6 and panitumumab chemotherapy in advanced stage rectal adenocarcinoma: a case report. World Journal of Surgical Oncology 2014 12:63.

\section{Submit your next manuscript to BioMed Central and take full advantage of:}

- Convenient online submission

- Thorough peer review

- No space constraints or color figure charges

- Immediate publication on acceptance

- Inclusion in PubMed, CAS, Scopus and Google Scholar

- Research which is freely available for redistribution 